\title{
Multi-Species Mean Field Spin Glasses. Rigorous Results
}

\author{
Adriano Barra, Pierluigi Contucci, \\ Emanuele Mingione and Daniele Tantari
}

\begin{abstract}
We study a multi-species spin glass system where the density of each species is kept fixed at increasing volumes. The model reduces to the Sherrington-Kirkpatrick one for the single species case. The existence of the thermodynamic limit is proved for all density values under a convexity condition on the interaction. The thermodynamic properties of the model are investigated and the annealed, the replica-symmetric and the replica symmetry breaking bounds are proved using Guerra's scheme. The annealed approximation is proved to be exact under a high-temperature condition. We show that the replica-symmetric solution has negative entropy at low temperatures. We study the properties of a suitably defined replica symmetry breaking solution and we optimize it within a novel ziggurat ansatz. The generalized order parameter is described by a Parisi-like partial differential equation.
\end{abstract}

\section{Introduction and Main Results}

In this paper, we introduce and study a multi-species mean field spin glass model, ${ }^{1}$ i.e., a system composed by spins belonging to a finite number of different species, where spin couples interact through a centered gaussian variable whose variance depends only on the species they belong to.

We rigorously prove bounds for the model pressure and control their properties in a region of convexity defined in terms of the variance matrix of the interactions, namely when the interactions within a group dominate the inter-group interactions. In particular, we prove the existence of the thermodynamic limit of the model, the correctness of the annealed solution in the

\footnotetext{
1 See also other works on extensions to multipartite of standard mean-field spin glasses $[5,7,19,20]$ or ferromagnets [8-10].
} 
high-temperature limit, and we perform an extension of the broken replica symmetry bounds developed by Guerra for the SK model [11] suitably adapted to this multi-species extension. In particular, we formulate a novel ziggurat ansatz for the broken replica phase which acts as a rigorous upper bound for the free energy. Before starting to explore the model, it may be worth stressing that, while we introduced the model and developed the Guerra's bound for the free energy, Panchenko [15] proved also the reverse bound; hence, the two papers as a whole confirm the correctness of our ansatz.

More specifically, the system is composed by a finite number $S$ of species indexed by $s \in \mathcal{S}$. For each $s \in \mathcal{S}$, consider the set $\Lambda_{N}^{(s)} \subset \mathbb{Z}$ such that

$$
\begin{gathered}
\Lambda_{N}^{(s)} \bigcap \Lambda_{N}^{(p)}=\emptyset, \quad \forall s \neq p \in \mathcal{S} \\
\left|\Lambda_{N}^{(s)}\right|=N^{(s)}, \\
N=\sum_{s \in \mathcal{S}} N^{(s)} .
\end{gathered}
$$

Consider a disordered model defined by a collection $\left(\sigma^{(s)}\right)_{s \in \mathcal{S}}$ of using variables, meaning that $\sigma_{i}^{(s)}= \pm 1$ for each $\forall s \in \mathcal{S}, i \in \Lambda_{N}^{(s)}$. We denote by $\Sigma_{N}$ the family of possible spin configurations $\sigma=\left\{\sigma_{i}^{(s)}\right\}_{s \in \mathcal{S}, i \in \Lambda_{N}^{(s)}}$, then we have that $\left|\Sigma_{N}\right|=2^{N}$. In the sequel, the following standard definitions of spin glass theory will be used.

For every $N \in \mathbb{N}$, let $\left\{H_{N}(\sigma)\right\}_{\sigma \in \Sigma_{N}}$ be a family of $2^{N}$ gaussian r.v. which represent the Hamiltonian of the system, defined by

$$
H_{N}(\sigma):=-\frac{1}{\sqrt{N}} \sum_{s, p \in \mathcal{S}} \sum_{i \in \Lambda_{N}^{(s)}} \sum_{j \in \Lambda_{N}^{(p)}} J_{i j}^{(s p)} \sigma_{i}^{(s)} \sigma_{j}^{(p)},
$$

where the J's are Gaussian i.i.d. r.v. such that for every $s, p, i, j$ we have that

$$
\mathbb{E}\left(J_{i j}^{(s p)}\right)=0
$$

and

with $\Delta_{s p}^{2}=\Delta_{p s}^{2}$.

$$
\mathbb{E}\left(J_{i j}^{(s p)} J_{i^{\prime} j^{\prime}}^{\left(s^{\prime} p^{\prime}\right)}\right)=\delta_{s s^{\prime}} \delta_{p p^{\prime}} \delta_{i i^{\prime}} \delta_{j j^{\prime}} \Delta_{s p}^{2},
$$

The covariance matrix of the system is

$$
C_{N}(\sigma, \tau):=\mathbb{E}\left(H_{N}(\sigma) H_{N}(\tau)\right)
$$

and, thanks to (1.5) and(1.6), a simple computation shows that

$$
C_{N}(\sigma, \tau)=\frac{1}{N} \sum_{s, p \in \mathcal{S}} \Delta_{s p}^{2}\left(\sum_{i \in \Lambda_{N}^{(s)}} \sigma_{i}^{(s)} \tau_{i}^{(s)}\right)\left(\sum_{j \in \Lambda_{N}^{(p)}} \sigma_{j}^{(p)} \tau_{j}^{(p)}\right) .
$$

To show explicitly the dependence trough the choice of the various sizes we can define for every $s \in \mathcal{S}$ the relative density

$$
\alpha_{N}^{(s)}:=\frac{N^{(s)}}{N},
$$


and the relative overlap

$$
q_{N}^{(s)}(\sigma, \tau)=\frac{1}{N^{(s)}} \sum_{i \in \Lambda_{N}^{(s)}} \sigma_{i}^{(s)} \tau_{i}^{(s)},
$$

then the covariance matrix can be write in the form

$$
C_{N}(\sigma, \tau)=N \sum_{s \in \mathcal{S}} \sum_{p \in \mathcal{S}} \Delta_{s p}^{2} \alpha_{N}^{(s)} \alpha_{N}^{(p)} q_{N}^{(s)}(\sigma, \tau) q_{N}^{(p)}(\sigma, \tau) .
$$

We can introduce in a natural way a vector notation ( see Appendix A for a detailed explanation), and rewrite (1.11) as

$$
C_{N}(\sigma, \tau)=N\left(\mathbf{q}_{N}, \boldsymbol{\Delta} \mathbf{q}_{N}\right)
$$

where

$$
\mathbf{q}_{N}=\left(q_{N}^{(s)}(\sigma, \tau)\right)_{s \in \mathcal{S}}
$$

Example. For example, in the case of two species, namely $\mathcal{S}=\{a, b\}$, the covariance matrix $\mathbf{q}_{N}$ is a two-dimensional vector and $\boldsymbol{\Delta}$ is a $2 \times 2$ matrix defined by the entries

$$
\left[\begin{array}{cc}
\alpha_{N}^{(a)} \alpha_{N}^{(a)} \Delta_{a a}^{2} & \alpha_{N}^{(a)} \alpha_{N}^{(b)} \Delta_{a b}^{2} \\
\alpha_{N}^{(a)} \alpha_{N}^{(b)} \Delta_{a b}^{2} & \alpha_{N}^{(b)} \alpha_{N}^{(b)} \Delta_{b b}^{2}
\end{array}\right]
$$

The random partition function extends standard partition function for disordered systems and reads off as

$$
Z_{N}:=\sum_{\sigma} a_{N}(\sigma, \mathbf{h}) \mathrm{e}^{-H_{N}(\sigma)},
$$

where

$$
a_{N}(\sigma, \mathbf{h}):=\exp \left(\sum_{s \in \mathcal{A}} h^{(s)} \sum_{i \in \Lambda_{N}^{(s)}} \sigma^{(s)}\right),
$$

and $\mathbf{h}:=\left(h^{(s)}\right)_{s \in \mathcal{A}}$ is a vector which represents an external (magnetic in the physical literature) field acting in each party separately.

Remark 1.1. Notice that, to lighten the notation, in the l.h.s. of (1.14), and in the rest of the paper, we do not write explicitly the dependence on $\mathbf{h}$. With the same aim, the physical inverse temperature $\beta$, which appears in the standard definition of the partition function, in our case is set equal to 1 with no loss of generality as it can be recovered in every moment simply by properly rescaling the interactions parameters.

The main thermodynamic observable is the quenched pressure density, defined as

$$
p_{N}:=\frac{1}{N} P_{N}:=\frac{1}{N} \mathbb{E} \log Z_{N}
$$

It's useful also to introduce a basic object in spin glass theory, the so-called quenched Gibbs measure. 
We consider $n$ copies of the configuration space, denoted by $\sigma^{1}, \ldots, \sigma^{n}$ and, for every bounded function $f:\left(\sigma^{1}, \ldots, \sigma^{n}\right) \rightarrow \mathbb{R}$, we call the random $n$-Gibbs state the following r.v.

$$
\Omega_{N}(f):=\sum_{\sigma^{1}, \ldots, \sigma^{n}} f\left(\sigma^{1}, \ldots, \sigma^{n}\right) G_{N}\left(\sigma^{1}\right) \ldots G_{N}\left(\sigma^{n}\right)
$$

where

$$
G_{N}(\sigma):=\frac{a_{N}(\sigma, \mathbf{h}) \mathrm{e}^{-H_{N}(\sigma)}}{Z_{N}}
$$

is called Gibbs measure. Lastly we define the quenched Gibbs measure as

$$
\langle f\rangle_{N}:=\mathbb{E} \Omega_{N}(f) \text {. }
$$

First at all we prove, under a suitable condition, the existence of the thermodynamic limit for the quenched pressure density (1.16) when the species densities are kept constant, i.e., the limit of $N \rightarrow \infty$ is defined such that $\forall s \in \mathcal{S}$ the quantity $\alpha_{N}^{(s)}=\frac{N^{(s)}}{N}=\alpha^{(s)}$ is independent of $N$. More precisely, we prove the following

Theorem 1.2. If the matrix $\boldsymbol{\Delta}$ is positive semi-definite, then

$$
\lim _{N \rightarrow \infty} p_{N}=\sup _{N} p_{N}
$$

where the limit is taken at fixed densities.

Notice that, since the relative densities are kept constants, the condition that $\boldsymbol{\Delta}$ is positive semi-definite is independent of the $\alpha$ 's.

In the single species case, the model reduces to the SherringhtonKirkpatrick model. Previous work [11], by using the interpolation method, shows that the celebrated Parisi's solution is an upper bound for pressure. The interpolation (usually called RSB interpolation) is defined through a nondecreasing, piecewise constant function $x(q):[0,1] \longrightarrow[0,1]$ which represents the cumulative distribution of the overlap (see [17]) and the order parameter of the model. One of the key points of the proof is that the function $x(q)$ intrinsically defines a non-decreasing sequence $\left(m_{l}\right)_{l=0, \ldots, K+1}$, thus enabling the control of the sign of the derivative of the interpolating functional. Following the same approach, in the multi-species case, we define the order parameter as a suitable piecewise constant, right continuous, function

$$
x(\mathbf{u}):[0,1]^{S} \rightarrow[0,1] .
$$

Let us show the explicit construction. Let $K$ be an integer and consider a sequence of points in $\Gamma \in[0,1]^{S}$, with

$$
\Gamma:=\left(\mathbf{q}_{l}\right)_{l=0, \ldots, K}=\left(q_{l}^{(s)}\right)_{s \in \mathcal{S}, l=0, \ldots, K}
$$

such that for each $s \in \mathcal{S}$, we have

$$
0=q_{0}^{(s)} \leq q_{1}^{(s)} \leq \cdots \leq q_{K-1}^{(s)} \leq q_{K}^{(s)}=1 .
$$

Roughly speaking $\Gamma$ defines a path with $K$ steps in $[0,1]^{S}$ which is nondecreasing in each direction. 
We consider a sequence $\left(m_{0}, \ldots, m_{K+1}\right)$ such that $0=m_{0} \leq m_{1} \leq \cdots \leq$ $m_{K} \leq m_{K+1}=1$.

If we denote by $\theta(\cdot)$ the right continuous Heaviside function, we define the functional order parameter as

$$
x(\mathbf{u}):=\sum_{l=0}^{K}\left(m_{l+1}-m_{l}\right) \prod_{s \in \mathcal{S}} \theta\left(u^{(s)}-q_{l}^{(s)}\right)
$$

where $\mathbf{u}=\left(u^{(s)}\right)_{s \in \mathcal{S}}$ is vector in $[0,1]^{S}$.

The function $x$ defines an $S$-dimensional shape, that in the case of $S=2$, looks like a ziggurat ${ }^{2}$ and clearly, in the analogy of the single species case, it must represent the joint cumulative distribution of the relatives overlaps.

It is useful to introduce, for each $s \in \mathcal{S}$, the canonical projection operator $\mathcal{P}_{s}$ in $\mathbb{R}^{S}$, and for $l=0, \ldots, K$, the following quantity:

$$
Q_{l}^{(s)}=\frac{2}{\alpha^{(s)}} \mathcal{P}_{s}\left(\Delta \mathbf{q}_{l}\right)
$$

where $\mathcal{P}_{s}$ is the canonical projection operator in $\mathbb{R}^{S}$ define in (A.7).

Notice that, for each $s \in \mathcal{S}$, the sequence $\left(Q_{l}^{(s)}\right)_{l=0, \ldots K}$ is non-decreasing. To complete the picture, we need to introduce a transformed order parameter

$$
x_{\Delta}(\mathbf{u}):=\sum_{l=0}^{K}\left(m_{l+1}-m_{l}\right) \prod_{s \in \mathcal{S}} \theta\left(u^{(s)}-Q_{l}^{(s)}\right)
$$

defined for $\mathbf{u} \in \times_{s \in \mathcal{S}}\left[0, Q_{K}^{(s)}\right]$.

We define the trial RSB pressure as

$$
p_{\mathrm{RSB}}(x):=\log 2+\sum_{s \in \mathcal{S}} \alpha(s) f^{(s)}\left(0, h^{(s)}\right)-\frac{1}{2} \int_{\widetilde{\Gamma}} x(\mathbf{u}) \nabla_{\mathbf{u}}(\mathbf{u}, \boldsymbol{\Delta} \mathbf{u}) \cdot \mathrm{d} \mathbf{u}
$$

where, for each $s \in \mathcal{S}, f^{(s)}\left(u^{(s)}, y\right)$ is the solution of the following Parisi's PDE

$$
\frac{\partial f^{(s)}}{\partial u^{(s)}}+\frac{1}{2} \frac{\partial^{2} f^{(s)}}{\partial y^{2}}+\frac{1}{2} x_{\Delta}\left(u^{(s)}\right)\left(\frac{\partial f^{(s)}}{\partial y}\right)^{2}=0
$$

where $x_{\boldsymbol{\Delta}}\left(u^{(s)}\right)$ is the marginal value of the transformed order parameter and the boundary condition is

$$
f^{(s)}\left(Q_{K}^{(s)}, y\right)=\log \cosh (y) .
$$

The integral in (1.25) is a line integral on an arbitrary path $\widetilde{\Gamma}$ in the plan $\mathbf{u}$, starting from $\mathbf{0}$ and ending in $\mathbf{1}$, such that all the points $\left(\mathbf{q}_{l}\right)_{l=0, \ldots, K}$ belong to $\Gamma$, in other words $\Gamma \subset \widetilde{\Gamma}$.

The main result of the paper is the next

\footnotetext{
${ }^{2}$ Ziggurats are pyramid-like structures found in the ancient Mesopotamian valley and western Iranian plateau.
} 
Theorem 1.3. The following sum rule holds

$$
p_{N}=p_{\mathrm{RSB}}(x)-\frac{1}{2} \sum_{l=0}^{K}\left(m_{l+1}-m_{l}\right) \int_{0}^{1} \mathrm{~d} t\left\langle\left(\mathbf{q}_{N}-\mathbf{q}_{l}\right), \boldsymbol{\Delta}\left(\mathbf{q}_{N}-\mathbf{q}_{l}\right)\right\rangle_{N, l, t} .
$$

Moreover, if the matrix $\boldsymbol{\Delta}$ is positive semi-definite, we have the following bound

$$
p_{N} \leq p_{\mathrm{RSB}}(x)
$$

and the optimization gives

$$
p_{N} \leq \inf _{x} p_{\mathrm{RSB}}(x) .
$$

We stress the fact that the ziggurat ansatz forces the joint overlap distribution to have a very special structure. In a recent paper [15], which appeared after this work, the author proved that the previous bound is exact, showing that this structure in fact encodes all the information of the model.

All the results are given under the hypothesis that the mutual interaction does not exceed a threshold where the strength of the off-diagonal terms prevails on the inter-party interactions. The latter is intrinsically different because the model approaches the Hopfield model for neural network $[2,4,6]$, on which we plan to report soon.

To frame properly the main result within a clear scenario for the multispecies mean field spin glass, the paper is organized as follows: In Sect. 2, we prove Theorem 1.2, i.e., the existence of thermodynamic limit. Section 3 studies the annealed region with the second moment method. Section 4 considers the replica-symmetric bound and shows that at low temperatures, it has a negative entropy. Finally, in Sect. 5, we give an extensive proof of Theorem 1.3.

\section{The Thermodynamical Limit at Fixed Densities}

In this section, we prove Theorem 1.2. The strategy of the proof follows classical Guerra-Toninelli arguments. Let us consider two non-interacting and i.i.d. copies of the original system defined by the Hamiltonian (1.4) of sizes, respectively, $N_{1}, N_{2}$. Clearly, this implies that we have to consider $\forall s \in \mathcal{S}$ the relative subsets $\Lambda_{N_{1}}^{(s)}, \Lambda_{N_{2}}^{(s)}$ defined by the Eqs. (1.1), (1.2), (1.3) and such that

$$
\begin{gathered}
\Lambda_{N_{1}}^{(s)} \cup \Lambda_{N_{2}}^{(s)}=\Lambda_{N}^{(s)}, \\
\Lambda_{N_{1}}^{(s)} \cap \Lambda_{N_{2}}^{(s)}=\emptyset, \\
\left|\Lambda_{N_{1}}^{(s)}\right|=N_{1}^{(s)}, \\
\left|\Lambda_{N_{2}}^{(s)}\right|=N_{2}^{(s)}, \\
N_{1}^{(s)}+N_{2}^{(s)}=N^{(s)} .
\end{gathered}
$$

More explicitly, we can define, $\forall s \in \mathcal{S}$, the following

$$
\begin{aligned}
& \Lambda_{N}^{(s)}=\left\{1, \ldots, N^{(s)}\right\}, \\
& \Lambda_{N_{1}}^{(s)}=\left\{1, \ldots, N_{1}^{(s)}\right\},
\end{aligned}
$$




$$
\Lambda_{N_{2}}^{(s)}=\left\{N_{1}^{(s)}+1, \ldots, N^{(s)}\right\} .
$$

Consider the following interpolating Hamiltonian

$$
H_{N}(\sigma, t)=\sqrt{t} H_{N}(\sigma)+\sqrt{1-t}\left(H_{N_{1}}(\sigma)+H_{N_{2}}(\sigma)\right)
$$

where

$$
\begin{aligned}
& H_{N_{1}}(\sigma)=-\frac{1}{\sqrt{N_{1}}} \sum_{s, p \in \mathcal{S}} \sum_{i \in \Lambda_{N_{1}}^{(s)}} \sum_{j \in \Lambda_{N_{1}}^{(p)}} J_{i j}^{\prime(s p)} \sigma_{i}^{(s)} \sigma_{j}^{(p)}, \\
& H_{N_{2}}(\sigma)=-\frac{1}{\sqrt{N_{2}}} \sum_{s, p \in \mathcal{S}} \sum_{i \in \Lambda_{N_{2}}^{(s)}} \sum_{j \in \Lambda_{N_{2}}^{(p)}} J_{i j}^{\prime \prime(s p)} \sigma_{i}^{(s)} \sigma_{j}^{(p)},
\end{aligned}
$$

and where $J_{i j}^{\prime(s p)}$ and $J_{i j}^{\prime \prime(s p)}$ are i.i.d. of $J_{i j}^{(s p)}$.

As usual we consider the interpolating pressure

$$
P_{N}(t)=\mathbb{E} \log Z_{N}(t)=\mathbb{E} \log \sum_{\sigma} a_{N}(\sigma, \mathbf{h}) \mathrm{e}^{-H_{N}(\sigma, t)},
$$

whose boundaries values are

$$
\begin{aligned}
& P_{N}(1) \equiv P_{N}, \\
& P_{N}(0) \equiv P_{N_{1}}+P_{N_{2}},
\end{aligned}
$$

since $\Sigma_{N}=\Sigma_{N_{1}} \cup \Sigma_{N_{2}}$ and $\Sigma_{N_{1}} \cap \Sigma_{N_{2}}=\emptyset$.

Proposition 2.1. The t-derivative of the interpolating pressure is

$$
\frac{\partial}{\partial t} P_{N}(t)=-\frac{N}{2} \mathbb{E} \Omega_{N, t}\left(Q_{N}\right),
$$

where

$$
Q_{N}(\sigma, \tau):=\left(\mathbf{q}_{N}, \boldsymbol{\Delta} \mathbf{q}_{N}\right)-\frac{N_{1}}{N}\left(\mathbf{q}_{N_{1}}, \Delta \mathbf{q}_{N_{1}}\right)-\frac{N_{2}}{N}\left(\mathbf{q}_{N_{2}}, \Delta \mathbf{q}_{N_{2}}\right),
$$

and the vectors $\mathbf{q}_{N_{1}}, \mathbf{q}_{N_{2}}$ are defined as in (1.13).

Proof. The computation of the $t$-derivative works essentially in the same way exploited in Proposition A.1 with the following identifications:

$$
i \rightarrow \sigma, \quad a_{i} \rightarrow a_{N}(\sigma, \mathbf{h}), \quad U_{i} \rightarrow H_{N}(\sigma), \quad \widetilde{U}_{i} \rightarrow H_{N_{1}}(\sigma)+H_{N_{2}}(\sigma)
$$

The key ingredient is that the diagonal term vanishes by the condition $N=$ $N_{1}+N_{2}$.

Combining the Fundamental Theorem of Calculus and the previous proposition we have that

$$
P_{N}-P_{N_{1}}-P_{N_{2}}=-\frac{N}{2} \int_{0}^{1} \mathrm{~d} t \mathbb{E} \Omega_{N, t}\left(Q_{N}\right) .
$$

To finish the proof is sufficient to show that 
Proposition 2.2. If the matrix $\boldsymbol{\Delta}$ is positive semi-definite, then

$$
Q_{N}(\sigma, \tau) \leq 0
$$

for every $\sigma, \tau$ and $N$.

Proof. First at all, we write some fundamental relations. hold

By definitions (1.10), (2.1), (2.2), (2.3) we have that $\forall s \in \mathcal{S}$ the following

$$
N^{(s)} q_{N}^{(s)}(\sigma, \tau)=\sum_{i=1}^{N^{(s)}} \sigma_{i}^{(s)} \tau_{i}^{(s)}=\sum_{i=1}^{N_{1}^{(s)}} \sigma_{i}^{(s)} \tau_{i}^{(s)}+\sum_{N^{(s)}+1}^{N^{(s)}} \sigma_{i}^{(s)} \tau_{i}^{(s)}
$$

then

$$
q_{N}^{(s)}(\sigma, \tau)=\frac{N_{1}^{(s)}}{N^{(s)}} q_{N_{1}}^{(s)}(\sigma, \tau)+\frac{N_{2}^{(s)}}{N^{(s)}} q_{N_{2}}^{(s)}(\sigma, \tau) .
$$

Now, we observe that the condition of fixed relatives densities implies that

$$
\frac{N_{1}^{(s)}}{N^{(s)}}=\frac{N_{1}^{(s)}}{N_{1}} \frac{N}{N^{(s)}} \frac{N_{1}}{N}=\frac{\alpha^{(s)}}{\alpha^{(s)}} \frac{N_{1}}{N}=\frac{N_{1}}{N},
$$

and in a similar fashion

$$
\frac{N_{2}^{(s)}}{N^{(s)}}=\frac{N_{2}}{N}
$$

then $\forall s \in \mathcal{S}$ the following holds

$$
q_{N}^{(s)}(\sigma, \tau)=\frac{N_{1}}{N} q_{N_{1}}^{(s)}(\sigma, \tau)+\frac{N_{2}}{N} q_{N_{2}}^{(s)}(\sigma, \tau) .
$$

In vector notation, we can write

$$
\mathbf{q}_{N}=\frac{N_{1}}{N} \mathbf{q}_{N_{1}}+\frac{N_{2}}{N} \mathbf{q}_{N_{2}} .
$$

It is easy to see that if $\boldsymbol{\Delta}$ is a positive semi-definite, real, symmetric matrix, hence the function

$$
\mathrm{x} \rightarrow(\mathrm{x}, \boldsymbol{\Delta x})
$$

defined for $\mathbf{x} \in \mathbb{R}^{S}$ is convex and the conclusion follows straightforwardly from the relation $(2.14)$.

The last proposition, combined with equation (2.4), gives immediately the super additivity property of the pressure. As a consequence, since the quenched pressure density is bounded from the annealed one (see the next section), then by Fakete's lemma, we get the statement of the theorem. 


\section{The Annealed Bound}

As a first analysis, we can study the annealed approximation for the pressure and investigate in which case it is exact. Using Jensen inequality and the concavity of the function $x \rightarrow \log (x)$ we define the annealed approximation as a bound, i.e.,

$$
p_{N}=\frac{1}{N} \mathbb{E} \log Z_{N} \leq \frac{1}{N} \log \mathbb{E} Z_{N}=p_{N}^{A} .
$$

We can easily write $p_{N}^{A}$ as

$$
\begin{aligned}
p_{N}^{A} & =\frac{1}{N} \log \sum_{\sigma} \mathbb{E}^{-H_{N}(\sigma)}=\frac{1}{N} \log \sum_{\sigma} \mathrm{e}^{\frac{1}{2} C_{N}(\sigma, \sigma)}=\frac{1}{N} \log \sum_{\sigma} \mathrm{e}^{\frac{N}{2}(\mathbf{1}, \boldsymbol{\Delta} \mathbf{1})} \\
& =\log 2+\frac{1}{2}(\mathbf{1}, \boldsymbol{\Delta} \mathbf{1}) .
\end{aligned}
$$

We define the ergodic regime as the region of the phase space in which

$$
\lim _{N \rightarrow \infty} \frac{1}{N} \mathbb{E} \log Z_{N}=\lim _{N \rightarrow \infty} \frac{1}{N} \log \mathbb{E} Z_{N}=p^{A}=\log 2+\frac{1}{2}(\mathbf{1}, \boldsymbol{\Delta} \mathbf{1}) .
$$

For this purpose, we can use a Borel-Cantelli argument to investigate the second moment, hence checking when

$$
\frac{\mathbb{E}\left(Z_{N}^{2}\right)}{\mathbb{E}^{2}\left(Z_{N}\right)} \leq C<\infty
$$

for some constant $C \in \mathbb{R}$, uniformly in $N$. Since

$$
\begin{aligned}
\mathbb{E}\left(Z_{N}^{2}\right) & =\mathbb{E} \sum_{\sigma, \tau} \mathrm{e}^{-H_{N}(\sigma)-H_{N}(\tau)}=\sum_{\sigma, \tau} \mathrm{e}^{\frac{1}{2} \mathbb{E}\left(H_{N}(\sigma)+H_{N}(\tau)\right)^{2}} \\
& =\sum_{\sigma, \tau} \mathrm{e}^{N\left((\mathbf{1}, \boldsymbol{\Delta} \mathbf{1})+\left(\mathbf{q}_{N}, \boldsymbol{\Delta} \mathbf{q}_{N}\right)\right)}=\mathbb{E}^{2}\left(Z_{N}\right) 2^{-2 N} \sum_{\sigma, \tau} \mathrm{e}^{N\left(\mathbf{q}_{N}, \boldsymbol{\Delta} \mathbf{q}_{N}\right)}
\end{aligned}
$$

and using the gauge transformation $\tau_{i}^{(s)} \rightarrow \sigma_{i}^{(s)} \tau_{i}^{(s)}$,

$$
\frac{\mathbb{E}\left(Z_{N}^{2}\right)}{\mathbb{E}^{2}\left(Z_{N}\right)}=2^{-2 N} \sum_{\sigma, \tau} \mathrm{e}^{N\left(\mathbf{m}_{N}(\tau), \Delta \mathbf{m}_{N}(\tau)\right)}=2^{-N} \sum_{\tau} \mathrm{e}^{N\left(\mathbf{m}_{N}(\tau), \Delta \mathbf{m}_{N}(\tau)\right)},
$$

where we define $\mathbf{m}_{N}(\tau)=\left(m_{N}^{(s)}(\tau)\right)_{s \in \mathcal{S}}$, with $m_{N}^{(s)}(\tau)=\frac{1}{N^{(s)}} \sum_{i=1}^{N^{(s)}} \tau_{i}^{(s)}$. If $\operatorname{det} \boldsymbol{\Delta}>0$ we can linearize the quadratic form with a gaussian integration

$$
\begin{aligned}
\frac{\mathbb{E}\left(Z_{N}^{2}\right)}{\mathbb{E}^{2}\left(Z_{N}\right)} & =\frac{2^{-N}}{\sqrt{\operatorname{det} \boldsymbol{\Delta}}} \int \frac{\mathrm{d} \mathbf{z}}{2 \pi} \mathrm{e}^{-\frac{1}{2}\left(\mathbf{z}, \boldsymbol{\Delta}^{-1} \mathbf{z}\right)} \sum_{\tau} \mathrm{e}^{\sqrt{2 N}\left(\mathbf{m}_{N}(\tau), \mathbf{z}\right)} \\
& =\frac{1}{\sqrt{\operatorname{det} \boldsymbol{\Delta}}} \int \frac{\mathrm{d} \mathbf{z}}{2 \pi} \mathrm{e}^{-\frac{1}{2}\left(\mathbf{z}, \boldsymbol{\Delta}^{-1} \mathbf{z}\right)} \prod_{s \in \mathcal{A}} \cosh ^{N^{(s)}}\left(\frac{\sqrt{2 N}}{N^{(s)}} z^{(s)}\right) \\
& \left.=\frac{1}{\sqrt{\operatorname{det}(\boldsymbol{\Delta})}} \int \frac{\mathrm{d} \mathbf{z}}{2 \pi} \mathrm{e}^{-\frac{1}{2}\left(\mathbf{z}, \boldsymbol{\Delta}^{-1}\right.} \mathbf{z}\right) \mathrm{e}^{\sum_{s \in \mathcal{S}} N^{(s)} \log \cosh \left(\frac{\sqrt{2 N}}{N^{(s)}} z^{(s)}\right)}
\end{aligned}
$$

and, using the inequality $\log \cosh (x) \leq \frac{x^{2}}{2}$, we obtain

$$
\frac{\mathbb{E}\left(Z_{N}^{2}\right)}{\mathbb{E}^{2}\left(Z_{N}\right)} \leq \frac{1}{\sqrt{\operatorname{det}(\boldsymbol{\Delta})}} \int \frac{\mathrm{d} \mathbf{z}}{2 \pi} \mathrm{e}^{-\frac{1}{2}(\mathbf{z}, \hat{\boldsymbol{\Delta}} \mathbf{z})},
$$


where we have defined

$$
\hat{\Delta}=\Delta^{-1}-2 \boldsymbol{\alpha}^{-1}
$$

and the diagonal matrix $\boldsymbol{\alpha}=\operatorname{diag}\left(\left\{\alpha^{(s)}\right\}_{s \in \mathcal{S}}\right)$. Thus, we have just proved the following

Theorem 3.1. In the convex region, defined as $\operatorname{det} \boldsymbol{\Delta}>0$, as soon as $\hat{\boldsymbol{\Delta}}$ is positively defined, the pressure of the model does coincide with the annealed approximation, i.e.,

$$
p=\lim _{N \rightarrow \infty} \frac{1}{N} \mathbb{E} \log Z_{N}=\lim _{N \rightarrow \infty} \frac{1}{N} \log \mathbb{E} Z_{N}=p^{A}=\log 2+\frac{1}{2}(\mathbf{1}, \boldsymbol{\Delta} \mathbf{1}) .
$$

Remark 3.2. Note that such a region does exist and can be viewed as an hightemperature region. The two regions $\operatorname{det} \boldsymbol{\Delta}>0$ and $\hat{\boldsymbol{\Delta}}>0$ have a nonzero measure intersection, because, while the first is a condition on the relative size of the covariances, the latter is related to their absolute amplitude. Indeed, once fixed $\boldsymbol{\alpha}$ and $\boldsymbol{\Delta}$ satisfying $\operatorname{det} \boldsymbol{\Delta}>0$, we can rescale all the covariances with a parameter $\beta$, which play the role of the inverse temperature of the system, i.e., $\Delta_{s s^{\prime}} \rightarrow \beta \Delta_{s s^{\prime}}, \forall s, s^{\prime} \in \mathcal{S}$, leaving the relative sizes unaltered and the condition $\operatorname{det} \boldsymbol{\Delta}>0$ is still satisfied, such that $\hat{\boldsymbol{\Delta}} \rightarrow \beta^{-S} \boldsymbol{\Delta}^{-1}-2 \boldsymbol{\alpha}^{-1}$ is positively defined for $\beta$ small enough. ${ }^{3}$

\section{The Replica-Symmetric Bound}

In this section, we specialize Theorem 1.3 in the simplest case to obtain the so called RS bound.

The underlying idea is to compare the overlap vector (1.13) with a trial vector,

$$
\mathbf{q}_{\text {trial }}:=\left(q^{(s)}\right)_{s \in \mathcal{S}} .
$$

We define the trial replica-symmetric solution as

$$
p_{\text {RS }}\left(\mathbf{q}_{\text {trial }}\right):=\log 2+\sum_{s \in \mathcal{S}} \alpha^{(s)} p^{(s)}\left(\mathbf{q}_{\text {trial }}\right)+\frac{1}{2}\left(\left(\mathbf{1}-\mathbf{q}_{\text {trial }}\right), \boldsymbol{\Delta}\left(\mathbf{1}-\mathbf{q}_{\text {trial }}\right)\right),
$$

where

$$
p^{(s)}\left(\mathbf{q}_{\text {trial }}\right):=\int \mathrm{d} \mu(z) \log \cosh \left(\sqrt{\frac{2}{\alpha^{(s)}} \mathcal{P}_{s}\left(\Delta \mathbf{q}_{\text {trial }}\right)} z+h^{(s)}\right),
$$

and

$$
z \sim \mathcal{N}(0,1)
$$

Setting $K=2, m_{1}=0, m_{2}=1$ and $\mathbf{q}_{1}=\mathbf{q}_{\text {trial }}$ in Theorem 1.3 we obtain the following:

\footnotetext{
${ }^{3}$ Since $\boldsymbol{\Delta}$ is positively defined then also $\boldsymbol{\Delta}^{-1}$. Defining $a=\max _{s} \alpha^{(s)}$ and $\rho$ the smallest eigenvalue of $\boldsymbol{\Delta}^{-1}$, then, for any non-null vector $z,(z, \hat{\boldsymbol{\Delta}} z) \geq\left(\beta^{-S} \rho-a\right)(z, z)>0$ if $\beta^{S}<\rho / a$.
} 
Proposition 4.1. The following sum rule holds

$$
p_{N}=p_{\text {RS }}\left(\mathbf{q}_{\text {trial }}\right)-\frac{1}{2} \int_{0}^{1} \mathbb{E} \Omega_{N, t}\left(\left(\mathbf{q}_{N}-\mathbf{q}_{\text {trial }}\right), \boldsymbol{\Delta}\left(\mathbf{q}_{N}-\mathbf{q}_{\text {trial }}\right)\right) .
$$

Moreover, if the matrix $\boldsymbol{\Delta}$ is positive semi-definite, then the following bound holds

$$
p_{N} \leq p_{\text {RS }}\left(\mathbf{q}_{\text {trial }}\right)
$$

whose optimization gives

$$
p_{N} \leq \inf _{\mathbf{q}_{\text {trial }}} p_{\text {RS }}\left(\mathbf{q}_{\text {trial }}\right) .
$$

The optimization of (4.6) on $\mathbf{q}_{\text {trial }}$, gives a system of $S$ coupled selfconsistent equations, i.e., $\forall p \in \mathcal{S}$

$$
\sum_{s \in \mathcal{S}} \boldsymbol{\Delta}_{p s}\left[\int \mathrm{d} \mu(z) \tanh ^{2}\left(\sqrt{\frac{2}{\alpha^{(s)}} \mathcal{P}_{s}\left(\boldsymbol{\Delta}_{\text {trial }}\right) z}\right)-q^{(s)}\right]=0,
$$

This system admits a unique solution as soon as $\operatorname{det}(\boldsymbol{\Delta}) \neq 0$, thus whenever $\operatorname{det}(\boldsymbol{\Delta})>0, p_{\mathrm{RS}}\left(\mathbf{q}_{\text {trial }}\right)$ has a minimum in $\mathbf{q}_{\text {trial }}=\overline{\mathbf{q}}$ satisfying $\forall s \in \mathcal{S}$

$$
\bar{q}^{(s)}=\int \mathrm{d} \mu(z) \tanh ^{2}\left(\sqrt{\frac{2}{\alpha^{(s)}} \mathcal{P}_{s}(\boldsymbol{\Delta} \overline{\mathbf{q}}) z}\right)=\left\langle\mathcal{P}_{s}(\mathbf{q})\right\rangle_{t=0}
$$

The last equalities can be easily checked thanks to the factorizability of the one-body problem at $t=0$. In other words, the value of $\mathbf{q}_{\text {trial }}$ minimizing the overlap' s fluctuations of the original model (at $t=1$ ) is just the overlap's mean of the interpolating one-body trial at $t=0$.

Let us show now how the replica-symmetric bound violate the entropy positivity at low temperatures. Mirroring the historical scenario for monopartite spin-glasses, we can easily check that the replica-symmetric expression for the pressure (4.6) is not the exact solution of the model in the low temperature region by studying the behavior of the entropy. We can define it as the non-negative quantity

$$
s(\boldsymbol{\Delta})=\lim _{N \rightarrow \infty} s_{N}(\boldsymbol{\Delta})=-\frac{1}{N} \mathbb{E} \sum_{\sigma} G_{N}(\sigma, \boldsymbol{\Delta}) \log \left(G_{N}(\sigma, \boldsymbol{\Delta})\right),
$$

where $G_{N}(\sigma, \boldsymbol{\Delta})=Z_{N}^{-1}(\boldsymbol{\Delta}) \mathrm{e}^{-H_{N}(\sigma, \boldsymbol{\Delta})}$ is the Boltzmann measure. Notice that, unlike before, we write explicitly the dependence on the matrix $\boldsymbol{\Delta}$. Since $s_{N}(\boldsymbol{\Delta})=p_{N}(\boldsymbol{\Delta})-\frac{1}{N}\langle H(\sigma)\rangle_{N}$, we can write

$$
s(\boldsymbol{\Delta})=p(\boldsymbol{\Delta})-\left.\frac{\mathrm{d}}{\mathrm{d} \lambda} p(\lambda \boldsymbol{\Delta})\right|_{\lambda=1} .
$$

Now, we can define $s_{\mathrm{RS}}(\boldsymbol{\Delta})=p_{\mathrm{RS}}-\left.\frac{\mathrm{d}}{\mathrm{d} \lambda} p_{\mathrm{RS}}(\lambda \boldsymbol{\Delta})\right|_{\lambda=1}$. We can easily show that if the amplitude of the covariances is large enough, $s_{\mathrm{RS}}(\boldsymbol{\Delta})$ is strictly negative. Indeed, we have the following 
Proposition 4.2. In the regime of large covariances (low temperatures), the $R S$-entropy is strictly negative, i.e.,

$$
\lim _{\beta \rightarrow+\infty} s_{\mathrm{RS}}(\beta \boldsymbol{\Delta})<0,
$$

for any choice of $\boldsymbol{\Delta}$ with $(\operatorname{det}(\boldsymbol{\Delta})>0)$ and $\boldsymbol{\alpha}$, where $\beta \in \mathbb{R}^{+}$plays the role of the inverse temperature.

Proof. Using its definition

$$
s_{\mathrm{RS}}(\beta \boldsymbol{\Delta})=p_{\mathrm{RS}}(\beta \boldsymbol{\Delta}, \overline{\mathbf{q}})-\left.\frac{\partial}{\partial \lambda} p_{\mathrm{RS}}(\lambda \beta \boldsymbol{\Delta}, \overline{\mathbf{q}})\right|_{\lambda=1} .
$$

We note that, using (4.8), in the limit $\beta \rightarrow+\infty$, the optimized order parameters $\overline{\mathbf{q}} \rightarrow \mathbf{1}$. Explicating the derivative, it is easy to see that

$$
\lim _{\beta \rightarrow+\infty} s_{\mathrm{RS}}(\beta \boldsymbol{\Delta})=\lim _{\beta \rightarrow+\infty}-\frac{\beta^{2}}{2}((\mathbf{1}-\overline{\mathbf{q}}), \boldsymbol{\Delta}(\mathbf{1}-\overline{\mathbf{q}})) \leq 0
$$

Finally, we can state that the limit is strictly negative, using again (4.8) and noting that

$$
\begin{aligned}
\lim _{\beta \rightarrow+\infty} \beta\left(1-\bar{q}_{(s)}\right) & =\lim _{\beta \rightarrow+\infty} \beta \int \mathrm{d} \mu(z)\left(1-\tanh ^{2}\left(\beta \sqrt{\frac{2}{\alpha^{(s)}} \mathcal{P}_{s}(\boldsymbol{\Delta} \overline{\mathbf{q}}) z}\right)\right) \\
& =\frac{\int \mathrm{d} \mu(z)|z|}{\sqrt{\frac{2}{\alpha^{(s)}} \mathcal{P}_{s}(\boldsymbol{\Delta} \mathbf{1})}}>0 .
\end{aligned}
$$

The existence of a negative RS-entropy regime is a clear signal that the model is not always replica symmetric (certainly it is $\mathrm{RS}$ inside the annealed region defined in Theorem 3.1), but there exists a region in which the pressure $p(\boldsymbol{\Delta})$ is strictly lower than its RS bound $p_{\mathrm{RS}}(\boldsymbol{\Delta})$.

\section{The Broken Replica Symmetry Bound}

In this section, we give a proof of Theorem 1.3. It is enough to show that (1.28) holds, then we have straightforward conclusions. The strategy is to apply the RSB interpolation scheme introduced in Appendix A.

We define the interpolating Hamiltonian as

$$
H_{N}(\sigma, t):=\sqrt{t} H_{N}(\sigma)+\sqrt{1-t} \sum_{l=1}^{K} H_{N}^{l}\left(\sigma, \mathbf{q}_{l}\right)
$$

with

$$
H_{N}^{l}\left(\sigma, \mathbf{q}_{l}\right):=\sum_{s \in \mathcal{S}} H_{N}^{l,(s)}\left(\sigma^{(s)}, \mathbf{q}_{l}\right)
$$


where $H_{N}(\sigma)$ is the original Hamiltonian and, for each $l, H_{N}^{l,(s)}\left(\sigma^{(s)}, \mathbf{q}_{l}\right)$ are two independent one-body interaction Hamiltonian, defined as

$$
H_{N}^{l,(s)}\left(\sigma^{(s)}, \mathbf{q}_{l}\right):=-\sqrt{2} \sqrt{\mathcal{P}_{s}\left(\boldsymbol{\Delta}\left(\mathbf{q}_{l}-\mathbf{q}_{l-1}\right)\right)} \frac{1}{\sqrt{\alpha^{(s)}}} \sum_{i \in \Lambda_{N}^{(s)}} J_{i}^{l,(s)} \sigma_{i}^{(s)}
$$

where the $J$ 's are Gaussian i.i.d. r.v., independent of the other r.v., such that for every $l, s$ and $i$ we have that

$$
\mathbb{E}\left(J_{i}^{l,(s)}\right)=0
$$

and

$$
\mathbb{E}\left(J_{i}^{l,(s)} J_{i^{\prime}}^{l^{\prime},\left(s^{\prime}\right)}\right)=\delta_{l l^{\prime}} \delta_{s s^{\prime}} \delta_{i i^{\prime}} .
$$

After simple computations, we get

$$
\mathbb{E}\left(H_{N}^{l,(s)}\left(\sigma^{(s)}, \mathbf{q}_{l}\right) H_{N}^{l^{\prime},\left(s^{\prime}\right)}\left(\tau^{\left(s^{\prime}\right)}, \mathbf{q}_{l}\right)\right)=\delta_{l l^{\prime}} \delta_{s s^{\prime}} 2 N \mathcal{P}_{s}\left(\boldsymbol{\Delta}\left(\mathbf{q}_{l}-\mathbf{q}_{l-1}\right)\right) \mathcal{P}_{s}\left(\mathbf{q}_{N}\right)
$$

and then by (A.8), the covariance matrix of the trial Hamiltonian becomes

$$
\mathbb{E}\left(H_{N}^{l}\left(\sigma, \mathbf{q}_{l}\right) H_{N}^{l^{\prime}}\left(\tau, \mathbf{q}_{l^{\prime}}\right)\right)=\delta_{l l^{\prime}} 2 N\left(\left(\mathbf{q}_{l}-\mathbf{q}_{l-1}\right), \mathbf{\Delta} \mathbf{q}_{N}\right) .
$$

Keeping in mind Proposition A.2, we introduce the RSB interpolation scheme with the following identifications:

$$
i \rightarrow \sigma, \quad a_{i} \rightarrow a_{N}(\sigma, \mathbf{h}), \quad U_{i} \rightarrow H_{N}(\sigma), \quad B_{i}^{l} \rightarrow H_{N}^{l}\left(\sigma, \mathbf{q}_{l}\right)
$$

and we define the interpolating pressure as

$$
p_{N}(t):=\frac{1}{N} \mathbb{E} \log Z_{0, N}(t) .
$$

It is easy to check that the boundary values of $p_{N}(t)$ are

$$
\begin{aligned}
& p_{N}(1)=p_{N}, \\
& p_{N}(0)=\log 2+\sum_{s \in \mathcal{S}} \alpha^{(s)} f^{(s)}\left(0, h^{(s)}\right),
\end{aligned}
$$

where $f^{(s)}\left(u^{(s)}, h^{(s)}\right)$ is the solution of the Parisi's PDE (1.26).

In order to apply the interpolation argument, we have to compute the $t$-derivative of the interpolating pressure. A simple application of Proposition A.2 leads to the following

$$
\frac{\partial}{\partial t} p_{N}(t)=-\frac{1}{2}(\mathbf{1}, \boldsymbol{\Delta} \mathbf{1})-\frac{1}{2} \sum_{l=0}^{K}\left(m_{l+1}-m_{l}\right)\left\langle\left(\mathbf{q}_{N}, \Delta \mathbf{q}_{N}\right)-2\left(\mathbf{q}_{N}, \Delta \mathbf{q}_{l}\right)\right\rangle_{N, l, t} .
$$

To complete the proof of the Theorem, we need the following equivalence

Proposition 5.1. The following representation holds

$$
-\frac{1}{2}(\mathbf{1}, \boldsymbol{\Delta} \mathbf{1})+\frac{1}{2} \sum_{l=0}^{K}\left(m_{l+1}-m_{l}\right)\left(\mathbf{q}_{l}, \boldsymbol{\Delta} \mathbf{q}_{l}\right)=-\frac{1}{2} \int_{\widetilde{\Gamma}} \mathrm{d} \mathbf{u} \quad x(\mathbf{u}) \nabla_{\mathbf{u}}(\mathbf{u}, \boldsymbol{\Delta} \mathbf{u}) \cdot \mathrm{d} \mathbf{u} .
$$


Proof. We can use the explicit definition of $x(\mathbf{u})$ given in (1.22) to check that

$$
-\frac{1}{2} \int_{\widetilde{\Gamma}} x(\mathbf{u}) \nabla_{\mathbf{u}}(\mathbf{u}, \boldsymbol{\Delta} \mathbf{u}) \cdot \mathrm{d} \mathbf{u}=-\frac{1}{2} \sum_{l=0}^{K}\left(m_{l+1}-m_{l}\right) \int_{\Gamma_{l}} \nabla_{\mathbf{u}}(\mathbf{u}, \boldsymbol{\Delta} \mathbf{u}) \cdot \mathrm{d} \mathbf{u}
$$

where $\Gamma_{l}$ is the result of the action of the $\theta$ 's on the path $\widetilde{\Gamma}$, that is his component between the points $\mathbf{q}_{l}$ and $\mathbf{1}$. By the Gradient's Theorem, the integral is path independent and is equal to the increment of the potential function, that is the desired result.

Finally, combining (5.7), (5.8), (5.9) and Proposition 5.1, the proof of (1.28) is a simple application of the fundamental theorem of calculus.

\section{Acknowledgements}

Authors are grateful to MiUR trough the FIRB grants number RBFR08EKEV and RBFR10N90W and PRIN grant number 2010HXAW77 and to Sapienza University of Rome and Alma Mater Studiorum, Bologna University. AB is partially funded by GNFM (Gruppo Nazionale per la Fisica Matematica) which is also acknowledged.

\section{Appendix A. Notation and Technical Tools}

Much of the recent progresses in the study of mean field spin glass models is based on methods and arguments introduced by Guerra in a series of works (see e.g., [3,11-14]), constituting the interpolation method. Beyond the original works, the interested reader can find a detailed and complete exposition with several applications of this method in [18], while in [1], these techniques are shown at work on the simpler Curie-Weiss model. In order to present a selfconsistent exposition hereafter, we outline briefly the basic ideas.

Let $N$ be an integer, and for $i \in I=\{1, \ldots, N\}$, let $U_{i}$ and $\widetilde{U}_{i}$ be two families of centered Gaussian random variables, independent each other, uniquely determined by the respective covariance matrices $\mathbb{E}\left(U_{i} U_{j}\right)=C_{i j}$ and $\mathbb{E}\left(\widetilde{U}_{i} \widetilde{U}_{j}\right)=\widetilde{C}_{i j}$. We treat the set of indices $i$ as configuration space for some statistical mechanics system. We define the Hamiltonian interpolating function as the following random variable

$$
H_{i}(t):=\sqrt{t} U_{i}+\sqrt{1-t} \tilde{U}_{i}
$$

where $t \in[0,1]$ is the real parameter used for interpolation.

Let us introduce the multi-species framework. Suppose that the system is composed by a finite number $S$ of species indexed by $s \in \mathcal{S}$, then $|\mathcal{S}|=S$. We assume that:

- the configuration space is decomposed in a disjoint union:

$$
I=\bigcup_{s \in \mathcal{S}} I^{(s)}
$$


- the U's are also decomposed in the following way:

$$
U_{i}=\sum_{s, p \in \mathcal{S}} U_{i}^{(s p)}
$$

where $U_{i}^{(s p)}$ is a family of gaussian r.v. such that the covariance matrix is of the form

$$
\mathbb{E}\left(U_{i}^{(s p)} U_{j}^{\left(s^{\prime} p^{\prime}\right)}\right)=\Delta_{s p}^{2} \delta_{s s^{\prime}} \delta_{p p^{\prime}} C_{i j}^{(s)} C_{i j}^{(p)}
$$

where $C_{i j}^{(s)}$ is a covariance matrix defined on $I^{(s)} \times I^{(s)}$.

Notice that the covariance matrix defined in (A.2) is the Schur-Hadamard product of the $C_{i j}^{(s)}$ and then is positive definite. The family of positive parameters $\left(\Delta_{s p}^{2}\right)_{s, p \in \mathcal{S}}$ tunes the interactions between the various species.

For a fixed couple $(i, j)$, we can think at each $C_{i j}^{(s)}$ as a component of a vector in the space $\mathbb{R}^{S}$, and then, thanks to (A.1) and (A.2), the covariance matrix of the entire system can be rewritten, with a slightly abuse of notation, as a quadratic form in $\mathbb{R}^{S}$, namely as

$$
C_{i j}=\mathbb{E}\left(U_{i} U_{j}\right)=\sum_{s, p \in \mathcal{S}} C_{i j}^{(s)} \Delta_{s p}^{2} C_{i j}^{(p)}=(\mathbf{C}, \Delta \mathbf{C}),
$$

where $\mathbf{C}:=\left(C_{i j}^{(s)}\right)_{s \in \mathcal{S}}$ is a vector in $\mathbb{R}^{S}$ and $\boldsymbol{\Delta}$ is the real-symmetric matrix defined by the entries

$$
\Delta:=\left(\Delta_{s p}^{2}\right)_{s, p \in \mathcal{S}} .
$$

Suppose for simplicity that $C_{i i}^{(s)}=\sqrt{c}$ for some $c \in \mathbb{R}^{+}$for each $i \in I, s \in \mathcal{S}$, that is

$$
C_{i i}=c(\mathbf{1}, \boldsymbol{\Delta} \mathbf{1})
$$

where

$$
\mathbf{1}:=(1)_{s \in \mathcal{S}} .
$$

We make the same assumptions for the $\widetilde{U}$ 's. An application of integration by part formula gives the following

Proposition A.1. Consider the functional

$$
\varphi(t):=\mathbb{E} \log Z(t)
$$

then for its t-derivative the following holds

$$
\varphi^{\prime}(t)=\frac{1}{2}(c-\widetilde{c})(\mathbf{1}, \boldsymbol{\Delta} \mathbf{1})-\frac{1}{2}\langle(\mathbf{C}, \boldsymbol{\Delta} \mathbf{C})-(\widetilde{\mathbf{C}}, \boldsymbol{\Delta} \widetilde{\mathbf{C}})\rangle_{t} .
$$

where \langle\rangle$_{t}$ is the quenched measure (1.19) induced by $H_{i}(t)$.

In order to separate the contribution of the various species, let us introduce the operator $\mathcal{P}_{s}$ as the canonical projector in $\mathbb{R}^{S}$.

For any $s \in \mathcal{S}$ and for any vector $\mathbf{u}=\left(u^{(s)}\right)_{s \in \mathcal{S}}$ in $\mathbb{R}^{S}$, we have that

$$
\mathcal{P}_{s}(\mathbf{u}):=u^{(s)} \text {. }
$$


Clearly, for two vectors $\mathbf{u}, \mathbf{v}$, the following relation holds

$$
(\mathbf{u}, \Delta \mathbf{v})=\sum_{s \in \mathcal{S}} \mathcal{P}_{s}(\mathbf{u}) \mathcal{P}_{s}(\Delta \mathbf{v})=\sum_{s \in \mathcal{S}} \mathcal{P}_{s}(\Delta \mathbf{u}) \mathcal{P}_{s}(\mathbf{v})
$$

We discuss now briefly the RSB Guerra's interpolation for multipartite systems in the same setting of the original work [11].

Let $K$ be an integer and consider an arbitrary sequence of points $\Gamma:=$ $\left(\mathbf{q}_{l}\right)_{l=1, \ldots, K} \in[0,1]^{S}$. For each triple $(l, i, s)$ with $l=1,2, \ldots, K, i \in I, s \in$ $\mathcal{S}$, let us introduce the family of centered Gaussian random variables $B_{i}^{l,(s)}$ independent from the $U_{i}$ and uniquely defined through the covariances

$$
\mathbb{E}\left(B_{i}^{l,(s)} B_{j}^{l^{\prime},\left(s^{\prime}\right)}\right)=\delta_{s s^{\prime}} \delta_{l l^{\prime}} \mathcal{P}_{s}\left(\Delta \mathbf{u}_{l}(\Gamma)\right) \mathcal{P}_{s}\left(\widetilde{\mathbf{C}}_{l}\right)
$$

where, for each value of $l$, the component of the vector $\widetilde{\mathbf{C}}_{l}=\left(\widetilde{C}_{l, i j}^{(s)}\right)_{s \in \mathcal{S}}$, are covariance matrix defined on $I^{(s)} \times I^{(s)}$ and $\mathbf{u}_{l}(\Gamma)$ is an arbitrary vector in $\mathbb{R}^{S}$ which depends on the choice of the sequence $\Gamma$.

Notice that (A.9) implies independence between two different $l^{(s)}, l^{\prime(s)}$ levels of symmetry breaking of each $s$-species. For each $l=1,2, \ldots, K$ and $i \in I$, we can define the following family of random variables

$$
B_{i}^{l}:=\sum_{s \in \mathcal{S}} B_{i}^{l,(s)}
$$

then by (A.8) we have that

$$
\mathbb{E}\left(B_{i}^{l} B_{j}^{l^{\prime}}\right)=\delta_{l l^{\prime}}\left(\mathbf{u}_{l}(\Gamma), \Delta \widetilde{\mathbf{C}}_{l}\right) .
$$

Suppose for simplicity that $\widetilde{C}_{l, i i}^{(s)}=1$ for each $l, i, s$, that is

$$
\mathbb{E}\left(B_{i}^{l} B_{i}^{l^{\prime}}\right)=\delta_{l l^{\prime}}\left(\mathbf{u}_{l}(\Gamma), \boldsymbol{\Delta} \mathbf{1}\right) .
$$

Let us introduce the following notations for the average with respect to $B_{i}^{l}, U_{i}$,

$$
\begin{aligned}
\mathbb{E}_{l}(\cdot) & =\int \prod_{i} \mathrm{~d} \mu\left(B_{i}^{l}\right)(\cdot) \quad \forall l=1, \ldots, K, \\
\mathrm{~d} \mu\left(B_{i}^{l}\right) & =\prod_{s \in \mathcal{A}} \mathrm{d} \mu\left(B_{i}^{l,(s)}\right), \\
\mathbb{E}_{0}(\cdot) & =\int \prod_{i} \mathrm{~d} \mu\left(U_{i}\right)(\cdot), \\
\mathbb{E}(\cdot) & =\mathbb{E}_{0} \mathbb{E}_{1} \ldots \mathbb{E}_{K}(\cdot) .
\end{aligned}
$$

Consider a non-decreasing sequence of non-negative real numbers $\left(m_{0}, m_{1}, \ldots\right.$, $\left.m_{K}, m_{K+1}\right)$ with $m_{0}=0, m_{K+1}=1$ and define recursively the following the random variables

$$
\begin{aligned}
Z_{K}(t) & :=\sum_{i} \omega_{i} \exp \left(\sqrt{t} U_{i}+\sqrt{1-t} \sum_{l=1}^{K} B_{i}^{l}\right), \\
Z_{l-1}^{m_{l}} & :=\mathbb{E}_{l}\left(Z_{l}^{m_{l}}\right)
\end{aligned}
$$


Proposition A.2. Consider the functional

$$
\varphi(t)=\mathbb{E}_{0} \log \left(Z_{0}(t)\right),
$$

then for its t-derivative the following relation holds

$$
\varphi^{\prime}(t)=\frac{1}{2}(\mathbf{1}, \boldsymbol{\Delta} \mathbf{1})-\sum_{l=1}^{K}\left(\mathbf{u}_{l}(\Gamma), \boldsymbol{\Delta} \mathbf{1}\right)-\frac{1}{2} \sum_{l=0}^{K}\left(m_{l+1}-m_{l}\right)\left\langle(\mathbf{C}, \boldsymbol{\Delta} \mathbf{C})-\widehat{B}^{l}\right\rangle_{l, t}
$$

where $\widehat{B}^{0}:=0, \widehat{B}^{l}:=\sum_{l^{\prime}=1}^{l}\left(\mathbf{u}_{l}(\Gamma), \Delta \widetilde{\mathbf{C}}_{l^{\prime}}\right)$ and \langle\rangle$_{l, t}$ is a suitable deformed quenched measure.

We notice that the previous proposition can be restated in the language of Ruelle Probability Cascades [16].

\section{References}

[1] Barra, A.: Irreducible free energy expansion and overlap locking in mean field spin glasses. J. Stat. Phys. 123(3), 601-614 (2006)

[2] Barra, A., Bernacchia, A., Santucci, E., Contucci, P.: On the equivalence of Hopfield networks and Boltzmann machines. Neural Netw. 34, 1-9 (2012)

[3] Barra, A., Di Biasio, A., Guerra, F.: Replica symmetry breaking in mean field spin glasses trough Hamilton-Jacobi technique. J. Stat. Mech. P09006 (2010)

[4] Barra, A., Genovese, G., Guerra, F.: The replica symmetric behavior of the analogical neural network. J. Stat. Phys. 140, 784-796 (2010)

[5] Barra, A., Genovese, G., Guerra, F.: Equilibrium statistical mechanics of bipartite spin systems. J.Phys. A 44(24), 245002 (2011)

[6] Barra, A., Genovese, G., Guerra, F., Tantari, D.: How glassy are neural networks? JSTAT 07, 07009 (2012)

[7] Bovier, A., Klimovsky, A.: The Aizenman-Sims-Starr and Guerra's schemes for the SK model with multidimensional spins Electron. J. Probab. 14(8), 161$241(2009)$

[8] Fedele, M., Contucci, P.: Scaling limits for multispecies statistical mechanics mean-field models. J. Stat. Phys. 144(6), 1186-1205 (2011)

[9] Fedele, M., Unguendoli, F.: Rigorous results on the bipartite mean-field model. J. Phys. A Math. Theor. 45, 3850-3860 (2012)

[10] Gallo, I., Contucci, P.: Bipartite mean field spin systems. Existence and solution. Math. Phys. E J. 14, 463 (2008)

[11] Guerra, F.: Broken replica symmetry bounds in the mean field spin glass model. Commun. Math. Phys. 233, 1-12 (2003)

[12] Guerra, F.: The cavity method in the mean field spin glass model. In: Albeverio, S., et al. (eds.) Advances in Dynamical Systems and Quantum Physics. Singapore (1995)

[13] Guerra, F., Toninelli, F.L.: The infinite volume limit in generalized mean field disordered models. Markov Proc. Relat. Fields 9, 195-207 (2003)

[14] Guerra, F., Toninelli, F.L.: The thermodynamic limit in mean field spin glass models. Commun. Math. Phys. 230, 71-79 (2002) 
[15] Panchenko, D.: The Free Energy in a Multi-Species Sherrington-Kirkpatrick Model. arXiv:1310.6679 (2013)

[16] Panchenko, D., Talagrand, M.: Guerra's Interpolation Using Derrida-Ruelle Cascades. arXiv:0708.3641 (2007)

[17] Talagrand, M.: Mean field models for spin glasses, volume II. In: Advanced Replica-Symmetry and Low Temperature. Ergebnisse der Mathematik und ihrer Grenzgebiete. 3. Folge A Series of Modern Surveys in Mathematics, vol. 55. Springer-Verlag, New York (2011)

[18] Talagrand, M.: Spin glasses: a challenge for mathematicians. Cavity and mean field models. Springer Verlag, New York (2003)

[19] Talagrand, M.: A general form of certain mean field models for spin glasses. Probab. Theory Relat. Fields 143(1-2), 97-111 (2009)

[20] Talagrand, M.: Mean field models for spin glasses: some obnoxious problems. In: Spin Glasses Lecture Notes in Mathematics, vol. 1900, Springer, Berlin (2007)

\section{Adriano Barra}

Dipartimento di Fisica

Sapienza Università di Roma

Piazzale Aldo Moro, 5

Rome, Italy

e-mail: adriano.barra@gmail.com

Daniele Tantari

Dipartimento di Matematica

Sapienza Università di Roma

Piazzale Aldo Moro, 5

Rome, Italy

e-mail: daniele.tnt@gmail.com

Pierluigi Contucci and Emanuele Mingione

Dipartimento di Matematica, Università di Bologna

Piazza di Porta San Donato, 5

Bologna, Italy

e-mail: pierluigi.contucci@unibo.it;

emanuele.mingione2@unibo.it

Communicated by Anton Bovier.

Received: July 31, 2013.

Accepted: April 5, 2014. 